Interactive comment on "Interhemispheric bias in earth's climate response to orbital forcing" by $R$. Roychowdhury and R. M. DeConto

R. Roychowdhury and R. M. DeConto

rajarshi.es@gmail.com

Received and published: 1 May 2016 


\title{
Response to Anonymous Referee \#2
}

Interactive

comment

\author{
May 1, 2016
}

We thank the two anonymous reviewers for providing thorough and constructive feedback on our manuscript. In the following section, we respond to the specific points raised by each reviewer:

\section{Major Comment \#1}

My major criticism is the way the discussion is presented on the $\mathrm{S}, \mathrm{J}$, and bËĘ indexes. These indexes are not easy to understand, because of the threshold cut off and the time accumulation, and therefore the mechanism for their response patterns are not always straightforward (e.g. Figs.3-6). At least, the authors should present and discuss the global climate response in terms of the basic variables, such as temperature, before discussing the corresponding threshold index, say, S. As it stands, it is very difficult to follow the discussion and understand the pattern of index response is. For example, in Fig.5, why the sign is negative over land, and why the sign is reversed over the ocean?

Printer-friendly version

Discussion paper

Author Response: Our primary motivation for working with the Total Integrated Summer Insolation (S) and Summer Energy $(\mathrm{J})$ metrics is that these are more robust indica- 
tors of the melting of ice-sheets, as compared to simpler indices like summer temperature or insolation intensity. The $\mathrm{S}$ and $\mathrm{J}$ indices take into account both the insolation intensity, as well as the duration of the melt season. Hence, a positive change in the $S$ or $\mathrm{J}$ index can mean an increase in insolation intensity, or an increase in melt season duration, or both (similarly, a decrease in S can mean a decrease in insolation intensity, or melt duration, or both). However, we agree that these indices may not be easy to understand, because of their non-linear response to insolation changes. To provide additional clarification, we will revise our paper to include a more thorough discussion in terms of the basic variables like summer temperature, which will be followed by our discussion in terms of $\mathrm{S}$ and $\mathrm{J}$ indexes.

In Fig 5., we plot the estimated 'Land Hemispheric Bias' in surface temperatures for present day continental geography and orbit. The negative sign over land (Northern Hemisphere) means that the Northern Hemisphere land surfaces have a lower surface temperature when compared to a symmetric earth, in which both hemispheres are land dominated. Similarly, the positive signs over water imply that the surface temperatures are higher when compared to a symmetric earth.

\section{Minor Comment \#1}

I don't like the word "bias" here. Bias, at least to a climatologist, usually implies some systematic error (from some truth). Here, the LHB really refers to the potential climate impact of land mass of each hemisphere, and there is no error involved. It is just some idealization. (save a serious comparison with paleo world). I think "impact" or "effect" or some other words, will be much better than "bias".

Printer-friendly version

Author Response: We agree that the word "bias" brings about different connotations here. We propose to remove the word "bias" all together, and replace "Land Hemispheric Bias" with "Land Asymmetry Effect' (LAE).

Interactive

comment

\section{C3}




\section{Minor Comment \#2}

The authors should highlight one serious caveat in their study, the slab ocean, which assumes a constant ocean heat transport such that the readers should realize the paper is studying an idealized land hemispheric effect (or bias if they call it) in an idealized coupled world. This is important for two reasons. First, the slab ocean works only for short time scales. For paleoclimate application (as the paper is intended for), however, it is the final long term impact that matters. The long term impact can depend critically on the ocean circulation and can differ dramatically from that derived from slab ocean model. Second, due to Bjerkness compensation, the ocean heat transport usually will change in response to climate forcing

Author Response: We agree with the reviewer here, this issue is addressed explicitly in two places in our paper. First, in the methods section where we state: "We use the latest (2012) version of the Global ENvironmental and Ecological Simulation of Interactive Systems (GENESIS) 3.0 GCM with a slab ocean component (Thompson and Pollard, 1997) rather than a full-depth dynamical ocean (Alder et al., 2011). The slab-ocean version of the GCM allows numerous simulations with idealized global geographies and greatly simplifies interpretations of the sensitivity tests by precluding complications associated with ocean model dependencies.".

At the end of the paper, we state: "Future work should include complimentary simulations with AOGCMs, to explore the potential modifying role of ocean dynamics on the LHB, not accounted for here."

Furthermore, the actual ocean heat transport in the slab component is not fixed, but changes relative to the land-ocean fraction in each band of latitude and as a function of the local temperature gradient. While slab-ocean GCMs clearly have limitations, the use of a fully coupled AOGCM would be computationally impractical for this exercise, and would add additional, likely model-dependent, complexities to this first analysis of the LHB issue.

Interactive comment
Printer-friendly version

Discussion paper 


\section{Minor Comment \#3}

Fig.2, caption: seems to be of wrong sign in (b), from low to high obliquity i.e. (b) is high obliquity - low obliquity. Please clarify. Partly, this reflects the lack of discussion mechanism of the pattern of the index as discussed above.

Author Response: We agree that our wording in the caption is confusing, and we will correct this in future. For example, for HIGH and LOW obliquity experiments, we run our GCM simulations with the highest and lowest obliquity values. In Fig $2 \mathrm{c}$ and $2 \mathrm{~d}$, we show the difference in summer energy $(\mathrm{J})$ between the HIGH and LOW obliquity experiments. HIGH Obliquity experiment has a higher Summer Energy (J) in the high latitudes than the LOW Obliquity experiment; hence our HIGH-LOW figure shows positive values in the high latitudes. This is a great point, and we will fix our wordings in the revised manuscript.

Interactive comment on Clim. Past Discuss., doi:10.5194/cp-2015-156, 2016. 